\title{
Queilitis actínica: protectores de rayos ultravioleta que previenen la progresión maligna. Reporte de un caso
}

\author{
Actinic cheilitis: ultraviolet rays protectors that prevent malignant progression. \\ Report of a case
}

\author{
Tomas Madrid Bellio' (D), Elio Jiménez Batista ${ }^{2}$, Jonathan Harris Ricardo ${ }^{3}$ (D) Martha Carmona Lorduy ${ }^{4}$ (D)
}

Citación: Madrid-Bellio T, Jiménez-Batista E, Harris-Ricardo J, Carmona-Lorduy M, Queilitis actínica: protectores de rayos ultravioleta que previenen la progresión maligna. Ustasalud 2021; 20: 51-55.

Licencia Creative Commons

(ㄷ) (1) (9) $\Theta$

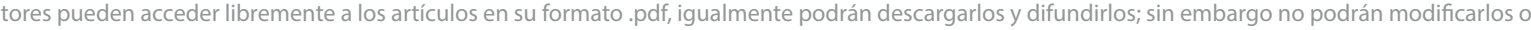
alterarlos, adicionalmente se debe reconocer la autoría de las personas que figuran en las publicaciones, pero estas no podrán ser comercializadas.

\section{Resumen}

La queilitis actínica es considerada un desorden potencialmente maligno, originado por la exposición excesiva y prolongada al sol, afecta el borde bermellón y la semimucosa labial, siendo la pérdida de definición entre el borde bermellón y la porción cutánea del labio, una característica clínica comúnmente encontrada. Puede presentarse de diversas formas, como placa blanca, erosión y úlceras. El grupo de riesgo está representado por personas entre la cuarta y sexta década de vida, con fototipo I y II de Fitzpatrick, dedicadas a trabajar al aire libre como agricultores, obreros, pescadores, etc. Histológicamente, la queilitis actínica presenta alteraciones epiteliales que pueden dar origen a un carcinoma de células escamosas, si el diagnóstico y tratamiento no son oportunos. El odontólogo es un pilar importante en el diagnóstico precoz y en la prevención de transformación maligna de esta patología, que cada vez es más común. Se presenta el reporte de un caso de un paciente masculino diagnosticado con queilitis actínica, se describen los aspectos clínicos, histopatológicos, factores de riesgo y manejo conservador con uso tópico de protector labial con factores de protección solar y evaluación clínica periódica para evitar la progresión maligna.

Palabras clave: Queilitis, neoplasias, rayos ultravioletas, diagnóstico, enfermedades de los labios.

\section{Abstract}

Actinic cheilitis is considered a potentially malignant disorder, caused by excessive and prolonged exposure to the sun, affects the vermilion border and the labial semi-mucosa, being the loss of definition between the vermilion border and the cutaneous portion of the lip, a commonly found clinical characteristic. It can present in a number of ways, including white plaque, erosion, and ulcers. The risk group is represented by people between the fourth and sixth decade of life, with Fitzpatrick phototype I and II, dedicated to working outdoors as farmers, laborers, fishermen, etc. Histologically, actinic cheilitis presents epithelial alterations that can give rise to squamous cell carcinoma, if diagnosis and treatment are not timely. The dentist is an important pillar in the early diagnosis and in the prevention of malignant transformation of this pathology, which is becoming more and more common. A case report of a male patient diagnosed with actinic cheilitis is presented, the clinical and histopathological aspects, risk factors and conservative management are described with topical use of lip balm with sun protection factors and periodic clinical evaluation, avoiding malignant progression.

Keywords: Cheilitis, neoplasms, ultraviolet rays, diagnosis, lip diseases.
1 Estudiante Facultad de Odontología, Universidad de Cartagena, Colombia.

2 Odontólogo. Especialista en Estomatologia y Cirugía Oral, Universidad de Cartagena. Colombia.

3 Odontólogo. Magíster en Microbiología Molecular, especialista en Estomatología y Cirugía Oral. Docente Corporación Universitaria Rafael Núñez y Universidad de Cartagena, Colombia.

4 Odontóloga. Magíster en Educación, especialista en Estomatología. Docente Universidad de Cartagena, Colombia.

\section{Autor de correspondencia:}

Tomas Madrid Bellio

Correo electrónico:

tmadridb1@unicartagena.edu.co 


\section{INTRODUCCIÓN}

La queilitis actínica (QA) es considerada una alteración potencialmente maligna, inducida principalmente por la exposición progresiva y excesiva a la luz ultravioleta (UV), estimada como el factor de riesgo ambiental más importante para el desarrollo de cáncer de piel [1,2]; estos rayos por su gran penetración afectan principalmente el labio inferior en un $95 \%$ de los casos, debido a que esta región anatómica está más expuesta y es la que recibe directamente la radiación $[3,4]$. Raramente se presenta en personas menores de 45 años, tiene predilección por el sexo masculino, con proporción hombre-mujer de 10:1, y en personas de piel blanca. La lesión tiene una evolución tan lenta que generalmente no es percibida por los pacientes, siendo reconocida como un hallazgo clínico durante la consulta odontológica [5]. Este desorden potencialmente maligno, tiene un riesgo de progresión en 10 años de un $6,1 \%$ a un $10,2 \%$ que se incrementa a un $40,0 \%$ en pacientes inmunocomprometidos [6].

Clínicamente se presenta con atrofia del borde bermellón del labio inferior, caracterizado por áreas de superficie lisa y placas blancas. La falta de definición entre el límite del borde bermellón y la piel es una de las características más prevalentes, a medida que la lesión avanza son observadas áreas ásperas cubiertas por tejido descamado, volviéndose más espesas y con una apariencia de una leucoplasia, especialmente cuando se extienden para la parte húmeda del labio [7].

Histológicamente, la lesión se caracteriza por la presencia de un epitelio pavimentoso estratificado atrófico, con grados variados de displasia, también puede ser observado infiltrado inflamatorio crónico subyacente al epitelio. El tejido conectivo se observa con regiones de alteración basofílica, amorfa y aceular, conocida como elastosis solar, siendo esta una alteración de las fibras colágenas y elásticas inducida por la exposición a los rayos UV [7]. El diagnóstico de la QA se obtiene por la correlación de los hallazgos clínicos y el estudio histopatológico $[8,9]$.

En las últimas décadas, el estudio de la fotocarcinogénesis se volvió un importante foco de investigación, principalmente en las áreas de dermatología y odontología, ya que, debido al deterioro de la capa de ozono y al aumento de las radiaciones UV, se ha incrementado el porcentaje de casos de neoplasias malignas de labio [5]. La radiación UV actúa como un carcinógeno mediante la inducción de daño celular causando mutaciones en el ADN y en genes supresores de tumores como el p53 [1,8].

El consumo de tabaco aumenta el riesgo de carcinoma en cavidad oral y más en aquellos que presentan QA. Eliminar el hábito de consumo de tabaco es fundamental para evitar la progresión maligna de la QA, este constituye un factor de riesgo elevado cuando interacciona con la exposición solar [4,8].

Debido al aumento en la prevalencia de cáncer de boca en el mundo, la QA merece especial cuidado debido a su potencial de malignización. Con el reporte del presente caso clínico, se destaca la importancia del papel del odontólogo en el diagnóstico precoz de esta patología, así como la importancia en la educación y la toma de medidas preventivas en pacientes que forman parte de la población de riesgo.

\section{PRESENTACIÓN DEL CASO}

Paciente masculino de 38 años, procedente de Cartagena, Colombia, que asiste a consulta estomatológica por presentar lesión blanquecina en el labio inferior, como antecedente importante el paciente manifiesta diagnóstico histopatológico de queilitis actínica desde hace dos años y trabajar como vendedor ambulante de playa, desde hace 14 años aproximadamente, manifestó que la lesión aumenta de tamaño con el pasar del tiempo y no utilizar barreras de protección solar.

En la exploración clínica presenta escala cromática de la piel de Fitzpatrick (IV), en el labio inferior del lado izquierdo se observó placa blanca, única, de forma rectangular, de bordes irregulares, de aproximadamente $1,5 \mathrm{~cm}$ de ancho x $1,0 \mathrm{~cm}$ de largo, seca, inmóvil y combinada con áreas ulceradas, descamativas y costrosas en todo el contorno y borde bermellón (Figura 1).

Se realizó biopsia incisional de la lesión, en la que se obtuvo muestra de tejido que abarcó semimucosa y mucosa labial. El estudio histopatológico reveló, epitelio con cambios displásicos moderados y presencia de elastosis solar con membrana basal preservada, compatible con la hipótesis de QA. En el control posquirúrgico realizado una semana después, se observó lesión 
en proceso de cicatrización sin signos de infección. Como conducta fue sugerida la aplicación tópica de hidratante labial con factores de protección solar (FPS), el uso de sombrero de ala ancha durante las jornadas laborales, así como el uso de sombrilla para evitar la exposición directa al sol.

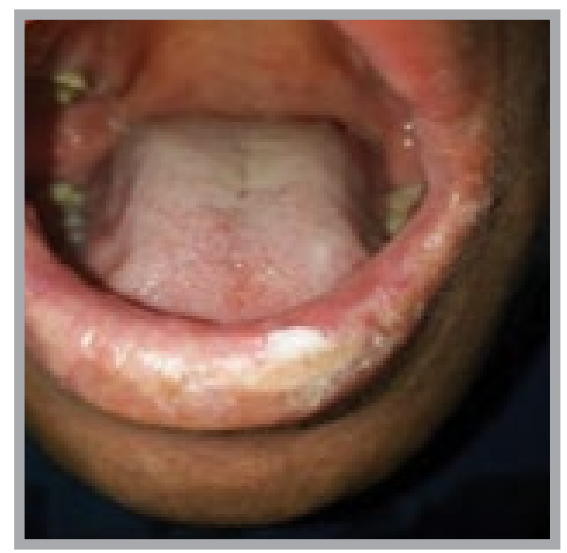

Figura 1. Placa blanca en semimucosa labial del lado izquierdo

Luego de 30 días, el paciente acude a consulta con lesión de aspecto más extendido y amplio (Figura 2). El crecimiento rápido de la lesión y la aparición repetitiva de esta en el labio inferior indica mayor actividad celular, signos que evidencian la falta de protección del labio ante la agresión que está recibiendo por el sol [8] dado que el paciente manifestó exposición solar con pocos cuidados protectores. Se decide hacer énfasis en las recomendaciones relacionadas con las barreras de protección solar anteriormente mencionadas, abandonar por lo pronto el trabajo de vendedor en la playa y control clínico en veinte días.

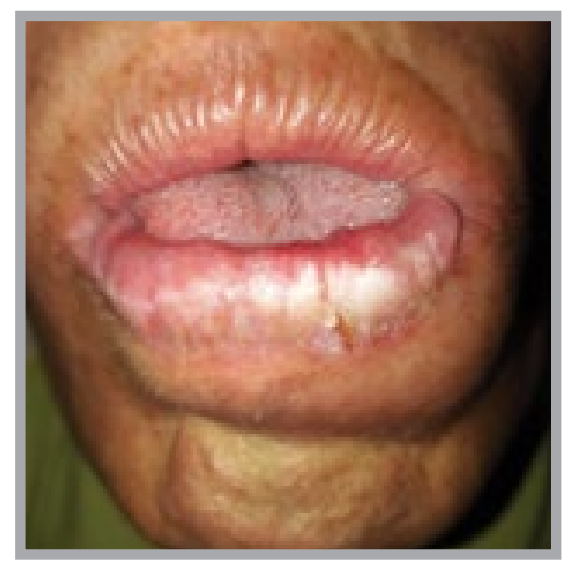

Figura 2. Control clínico a los treinta días con aumento en al tamaño de la lesión.
En el control clínico realizado a los cincuenta días, se observó evolución favorable del cuadro clínico, disminución del 90\% en el tamaño de la lesión, manchas color café, que indicaban cambios en la pigmentación y cicatriz del sitio de biopsia (Figura 3). El paciente destacó el uso continuo de las barreras de protección solar y poco tiempo de exposición al sol.

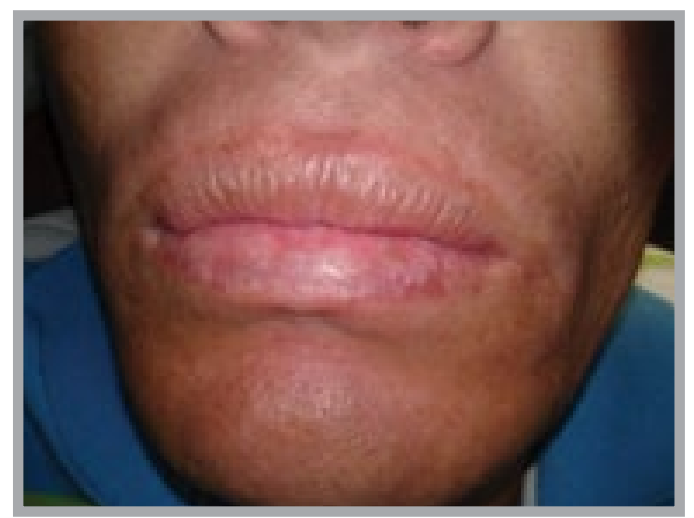

Figura 3. Control clínico a los cincuenta días con resolución de la lesión y cambios de pigmentación.

\section{DISCUSIÓN}

La QA es un tipo de inflamación de los labios causada por la exposición prolongada a la luz solar. Esta patología es un desorden potencialmente maligno que podría convertirse en carcinoma de células escamosas [10].

Con relación a los factores de riesgo Rodríguez-Blanco y colaboradores afirmaron que, entre los factores de riesgo significativos e independientes de la QA, el trabajo al aire libre o con exposición solar, antecedentes de cáncer y la edad ( $\geq 60$ años) son aspectos para considerar en la aparición de la patología [11]. Así mismo, de Souza y colaboradores evaluaron una población de 362 trabajadores en la playa, el 57,9\% estaba expuesto seis horas diarias al sol y el 15,5\% presentó QA [12]; coincidiendo con el presente caso en el que la condición laboral del paciente como vendedor ambulante en las playas por 14 años, sometido a largos períodos de exposición solar, fue un factor de riesgo determinante.

La ocupación laboral puede influir en la progresión de la QA, especialmente en aquellos que trabajan al aire libre, como pescadores, trabajadores de playas, jardineros, entre otros, porque están expuestos de forma constante y directa a la radiación UV. Los pacientes pueden 
reducir los riesgos de presentar lesiones más severas y la transformación de estas a malignas mediante el uso de bloqueador solar, ropa de protección UV, sombreros de ala ancha, bálsamos labiales con factores de protección solar o maquillaje que contengan protector solar y controles clínicos con el estomatólogo $[8,9]$.

El potencial oncogénico de la piel expuesta al sol de forma crónica es una preocupación importante. La posición anatómica y la forma prominente de los labios los hacen más susceptibles a la radiación UV, principalmente el labio inferior, las personas de piel más clara (fototipo I y II de Fitzpatrick), tienen menor actividad melanocítica y mayor absorción de radiación UV, aumentando así el riesgo de desarrollar lesiones potencialmente malignas como la QA y posteriormente el carcinoma de células escamosas de labio en algunos casos [12]; a pesar de que el color de piel del paciente era de fototipo IV de Fitzpatrick, lo que significa que es de bajo riego para desarrollar lesiones potencialmente malignas, el sometimiento a largos períodos de exposición solar fue el factor de riesgo determinante para el desarrollo de la QA.

Es difícil comparar la eficacia entre las numerosas opciones de tratamiento disponibles para la queilitis actínica. En muchos de los estudios publicados, los regímenes de tratamiento no son consistentes y con pocos reportes de controles clínicos; Carvalho y colaboradores realizaron una revisión de literatura sobre tratamiento quirúrgico versus no quirúrgico de la QA queilitis actínica, reportaron que es más favorable la terapéutica quirúrgica que la no quirúrgica, afirmaron que la recurrencia es menor cuando se aplica el tratamiento quirúrgico; sin embargo, concluyen que se necesitan más estudios que maximicen la estandarización metodológica y tengan un mayor rigor en el proceso de recolección de datos [13]. Lai y colaboradores revisaron literatura de la terapéutica empleada en la QA y destacaron la terapia con láser, terapia fotodinámica, imiquimod al 5\%, fluorouracilo, cirugía parcial, ácido tricloroacético al 50\% entre otros [14]. Se considera que la terapéutica seleccionada depende de las características clínicas e histopatológicas de cada paciente y que cada caso debe ser tratado individualmente.

Con respecto al papel del odontólogo frente a la QA en la práctica odontológica, Savage y colaboradores sugieren que el examen clínico de los labios y otras pa- tologías de los labios se conviertan en parte habitual del examen de rutina de los tejidos blandos que se realiza como parte del examen periódico odontológico [15]. Es importante destacar que el reconocimiento temprano de la QA puede permitir el desarrollo de estrategias que eviten su progresión.

Ríos y colaborabores, y Rodriguez-Blanco y colaboradores refieren que se deben realizar programas de promoción, prevención y diagnóstico temprano en poblaciones de riesgo, fomentando el autocuidado, utilizando medidas generales de cómo evitar la exposición solar, uso de protector solar y cubrir las áreas expuestas directas al sol $[16,17]$.

Es importante resaltar que las medidas de prevención, como: el uso de hidratantes labiales con FPS, el uso de sombreros de ala ancha o sombrilla, el uso de bloqueador solar y la reducción del número de horas de exposición al sol son esenciales para las personas que se encuentran en la población de riesgo, así como la concientización de estas en hacerse exámenes odontológicos periódicos con el fin de prevenir el aparecimiento y la progresión de la QA $[1,6]$.

\section{CONCLUSIÓN}

El uso de las barreras de protección solar en pacientes con QA como bálsamos labiales con protector solar, sombrillas, sombreros de ala ancha, disminuir el tiempo de exposición solar, entre otras, son medidas conservadoras que ayudan en la evolución positiva de la patología y evitan la progresión maligna.

\section{REFERENCIAS}

[1] Boza-Oreamuno Y, Guillén-Colombari D. Queilitis actínica: Reporte de tres casos y revisión de literatura. Odovtos. 2018; 20(3):33-42.

[2] Maia HC, Pinto NA, Pereira Jdos S, de Medeiros AM, da Silveira ÉJ, Miguel MC. Potentially malignant oral lesions: clinicopathological correlations. Einstein (Sao Paulo). 2016;14(1):35-40.

[3] Boza Y, Rojas I. Elastosis en la queilitis actínica. Revisión de literatura. Odovtos-Int J Dent Sc. 2018; 20(2):51-60. doi:10.15517/ijds.v0i0.32590

[4] Santos RFD, Oliveira RL, Gallottini M, Caliento R, Sarmento DJS. Prevalence of and factors associated with actinic cheilitis in extractive mining workers. Braz. Dent. J. 2018; 29(2):214-221. doi: 10.1590/0103-6440201801605. 
[5] de Oliveira Ribeiro A, da Silva LC, Martins-Filho PR. Prevalence of and risk factors for actinic cheilitis in Brazilian fishermen and women. Int J Dermatol. 2014;53(11):1370-6. doi: 10.1111/ijd.12526

[6] Boza OYV, Martínez A, Rojas IG. Evaluación histomorfométrica de la elastosis en queilitis actínica. Odovtos-Int J Dent Sc. 2016;18(3):51-59.

[7] Neville BW, Damm DD, Allen CM, Bouquot JE. Oral and Maxillofacial Pathology. 3rd edition. St. Louis: Saunders ELSEVIER; 2009.

[8] Hernández-Osorio C, Fuentes-Palma B, Cartes-Velásquez R. Queilitis actínica: aspectos histológicos, clínicos y epidemiológicos. Rev Cubana Estomatol. 2016;53(2):45-55.

[9] Orozco P, Vásquez S, Venegas B, Rivera C. Prevalencia de queilitis actínica en trabajadores expuestos a radiación ultravioleta en Talca, Chile. Rev. Clin. Periodoncia Implantol. Rehabil. Oral. 2013;6(3):127-129. doi:10.4067/S0719-01072013000300005.

[10] Mello FW, Melo G, Modolo F, Rivero ER. Actinic cheilitis and lip squamous cell carcinoma: Literature review and new data from Brazil. J Clin Exp Dent. 2019;11(1):62-69. doi:10.4317/jced.55133.

[11] Rodríguez-Blanco I, Flórez Á, Paredes-Suárez C, Rodríguez-Lojo R, González-Vilas D, Ramírez-Santos A, et al. Actinic cheilitis prevalence and risk factors: A cross-sectional, multicentre study in a population aged 45 years and over in north-west Spain. Acta Derm Venereol. 2018;98(10):970-974. doi:10.2340/00015555-3014.
[12] de Souza Lucena EE, Costa DC, da Silveira EJ, Lima KC. Prevalence and factors associated to actinic cheilitis in beach workers. Oral Dis. 2012;18(6):575-9. doi: 10.1111/j.1601-0825.2012.01910.x.

[13] Carvalho MV, de Moraes SLD, Lemos CAA, Santiago Júnior JF, Vasconcelos BCDE, Pellizzer EP. Surgical versus non-surgical treatment of actinic cheilitis: A systematic review and meta-analysis. Oral Dis. 2019;25(4):972-981. doi: 10.1111/odi.12916.

[14] Lai M, Pampena R, Cornacchia L, Pellacani G, Peris K, Longo C. Treatments of actinic cheilitis: A systematic review of the literature. J Am Acad Dermatol. 2020;83(3):876-887. doi: 10.1016/j.jaad.2019.07.106

[15] Savage NW, McKay C, Faulkner C. Actinic cheilitis in dental practice. Aust Dent J. 2010;55 Suppl 1:78-84. doi:10.1111/j.1834-7819.2010.01202.

[16] Ríos P, Maldonado C, Norambuena P, Donoso M. Prevalencia de queilitis actínica en pescadores artesanales, Valdivia, Chile. Int. J. Odontostomat. 2017;11(2):192197. DOI:10.4067/S0718-381X2017000200012.

[17] Rodriguez-Blanco I, Florez Á, Paredes-Suárez C, Rodríguez-Lojo R, González-Vilas D, Ramírez-Santos A, et al. Use of lip photoprotection in patients suffering from actinic cheilitis. Eur J Dermatol. 2019;29(4):383-386. doi:10.1684/ejd.2019.3604.

\section{Correo electrónico de los autores:}

Tomas Madrid Bellio: tmadridb1@unicartagena.edu.co Elio Jiménez Batista: eliojb90@gmail.com Jonathan Harris Ricardo: j.harris.r@hotmail.com Martha Carmona Lorduy: mcarmonal@unicartagena.edu.co 